PPPL-2866

UC-420,427

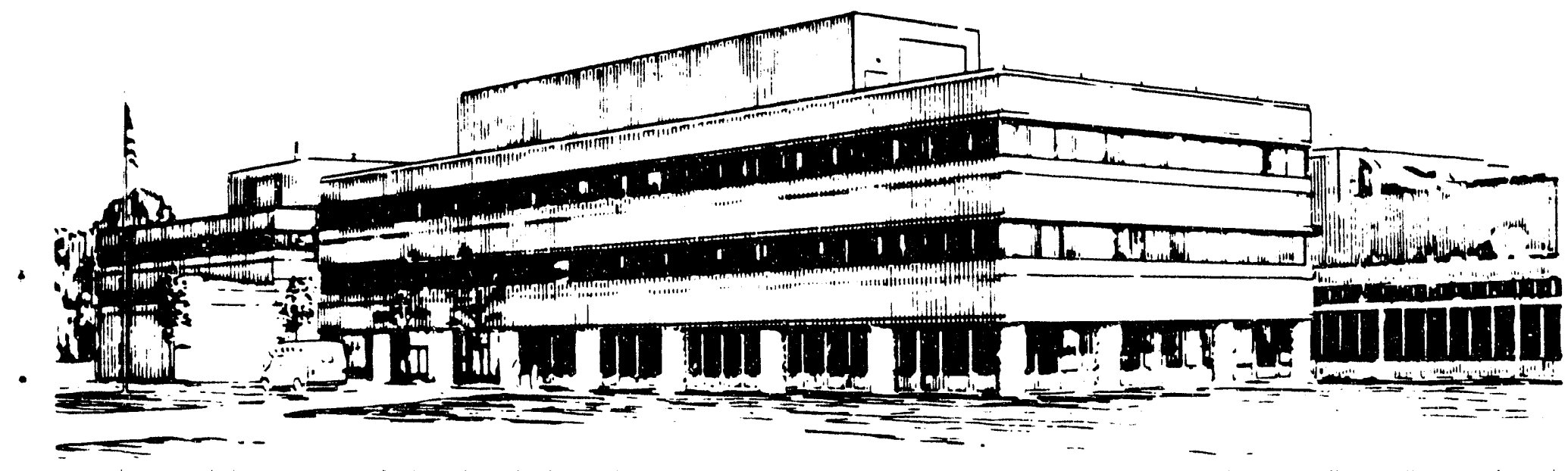




\section{NOTICE}

This report was prepared as an account of work sponsored by an agency of the United States Government. Neither the United States Government nor any agency thereof, nor any of their employees, makes any warranty, express or implied, or assumes any legal liability or responsibility for the accuracy, completeness, or usefulness of any information, apparatus, product, or process disclosed, or represents that its use would not infringe privately owned rights. Reference herein to any specific commercial produce, process, or service by trade name, trademark, manufacturer, or otherwise, does not necessarily constitute or imply its endorsement, recommendation, or favoring by the United States Government or any agency thereof. The views and opinions of authors expressed herein do not necessarily state or reflect those of the United States Government or any agency thereof.

\section{NOTICE}

This report has been reproduced from the best available copy.

Available in paper copy and microfiche.

Number of pages in this report: 19

DOE and DOE contractors can obtain copies of this report from:

Office of Scientific and Technical Information P.O. Box 62

Oak Ridge, TN 37831;

(615) $576-8401$.

This report is publicly available from the:

National Technical Information Service

Department of Commerce

5285 Port Royal Road

Springfield, Virginia 22161

(703) $487-4650$ 


\title{
Neoclassical Transport in High $\beta$ Tokamaks
}

\author{
S.C. Cowley \\ Princeton Plasma Physics Laboratory \\ P.O. Box 451, Princeton, New Jersey, 08543
}

\begin{abstract}
Neoclassical, transport in high $\beta$ large aspect ratio tokamaks is calculated. The variational method introduced by Rosenbluth, et al., [Phys. Fluids, 15, 116(1972)] is used to calculate the full Onsager matrix in the banana regime. These results are part of a continuing study of the high $\beta$ large aspect ratio equilibria introduced in Cowley, et al., [Phys. Fluids B, 3, 2069(1991)]. All the neoclassical coefficients are reduced from their nominal low $\beta$ values by a factor $\left(\epsilon / q^{2} \beta\right)^{\frac{1}{2}}$. This factor is the ratio of plasma volume in the boundary layer to the volume in the core. The fraction of trapped particles on a given flux surface $\left\langle f_{t}\right\rangle$ is also reduced by this factor so that $\left\langle f_{t}\right\rangle \sim \epsilon^{1 / 2}\left(\epsilon / q^{2} \beta\right)^{\frac{1}{2}}$. Special attention is given to the current equation, since this is thought to be relevant at low $\beta$ and therefore may also be relevant at high $\beta$. The bootstrap current term is found to exceed the actual current by a factor of the square root of the aspect ratio.
\end{abstract}




\section{Introduction}

This paper is the third in a series of papers investigating high $\beta$ tokamaks. We are, of course, concerned with the attractiveness of high $\beta$ tokamaks as fusion devices. In the first paper, ${ }^{1}$ which is refered to here as $C K^{3}$, we constructed the equilibrium of high $\beta$ large aspect ratio tokamaks. In the second paper, ${ }^{2}$ local MHD stability at high $\beta$, i.e., stability to interchange and ballooning modes was demonstrated. In the present paper, the particle orbits and the collisional transport, the so-called neoclassical transport, of a high $\beta$ tokamak are calculated. One would be extremely gratified but surprised if high $\beta$ tokamaks are governed by neoclassical crossfield transport of particles and heat. Surprised, because low $\beta$ tokamaks have anomalously large crossfield transport which is thought to be caused by turbulence. On the other hand experiments indicate that the neoclassical parallel current equation, involving bootstrap current and ohmic electric field current drive, is describing correctly the current evolution at low $\beta$. There is, therefore, some hope that at high $\beta$ the neoclassical parallel current equation is relevant. The orbit quantities derived in this paper are useful for future studies of the kinetic stability properties.

As we shall show, neoclassical transport coefficients at high $\beta$ are reduced from those at low $\beta$. This reduction is due (in the banana regime) to the reduction in the fraction of trapped particles on a flux surface, $\left\langle f_{t}\right\rangle$. Both the neoclassical transport coefficients and $\left\langle f_{t}\right\rangle$ are reduced by the factor $\left(\epsilon / q^{2} \beta\right)^{\frac{1}{2}}$ which is small at high $\beta$. This reduction is easily understord from the equilibrium properties. The equilibrium consists of a core region and a boundary layer (see Fig. 2). In the core region the flux surfaces are straight and functions of the major radius alone. Thus, $|\mathbf{B}|$ is constant on the flux surface in the core. The boundary layer follows the boundary and $|\mathbf{B}|$ varies over a flux surface by a factor $\epsilon$ (where $\epsilon=a / R_{0}$ is the inverse aspect ratio, see Fig. 1). The fraction of volume occupied by the boundary layer is $\left(\epsilon / q^{2} \beta\right)^{\frac{1}{2}}$. There are no trapped particles in the core and trapped particles are a fraction $\epsilon^{1 / 2}$ of the particles in the boundary layer. Thus, the fraction of the particles on a given surface that are trapped, $\left\langle f_{t}\right\rangle$, is given by $\left\langle f_{t}\right\rangle \sim \epsilon^{\frac{1}{2}}\left(\epsilon / q^{2} \beta\right)^{\frac{1}{2}}$. Passing particles spend a fraction $\left(\epsilon / q^{2} \beta\right)^{\frac{1}{2}}$ of their time in the boundary layer. Since they do not drift relative to the flux surface in the core their transport is also weighted by the factor $\left(\epsilon / q^{2} \beta\right)^{\frac{1}{2}}$.

Since the bootstrap current exceeds the actual current by a factor $\epsilon^{-1 / 2}$, the electric field primarily balances the bootstrap current. Accordingly the electric field is the opposite direction to the parallel current. At the magnetic axis, the bootstrap current is zero and the electric field balances the actual current. The resistive evolution is therefore towards a hollow q profile, with $q$ rising on axis and falling off axis. The solution of the flux diffusion equation is not attempted here.

In Sec. II we review the equilibrium calculation which is given in detail in $C K^{3}$. Also in this section, we calculate $|\mathbf{B}|$ since it is important for neoclassical theory. Orbit properties 
are discussed in Sec. III along with a heuristic derivation of banana transport at high $\beta$. In Sec. IV, we state the results of the formal neoclassical theory. The derivation follows the low $\beta$ large aspect ratio calculation given in Hinton and Hazeltine's review article ${ }^{3}$ we, therefore, omit the derivation. The only detail of the derivation needing clarification is the calculation of the pitch angle and flux surface integrals - these details are given in the Appendix.

\section{Summary of the Equilibrium}

In this section we summarize the relevant features of the equilibrium solution given in $C K^{3}$. We shall not give the details of the derivation here. The magnetic field is given by the usual form,

$$
\mathrm{B}=\frac{1}{R} \nabla \psi \times \mathrm{e}_{\phi}+\frac{F}{R} \mathbf{e}_{\phi},
$$

where $\mathrm{e}_{\phi}$ is a unit vector in the toroidal direction and $R$ is the distance from the symmetry axis, see Fig. 1. The safety factor, $q(\psi)$, is given by

$$
q(\psi)=\frac{F(\psi)}{2 \pi} \oint \frac{d l_{p}}{R|\nabla \psi|},
$$

where $d l_{p}$ is a poloidal line element. The integration is performed along poloidal projections of surfaces of constant $\psi$. The equilibrium is taken to be bounded by a conducting wall of arbitrary shape but large aspect ratio, i.e., $R \gg a$. The geometry is given in Fig.1. Note that noncircular boundaries are considered in Sec. VI of $C K^{3}$ as a simple generalization of the circular case. We shall use the same normalizations as $C K^{3}$. These normalizations are chosen to reflect the ordering $\beta \sim O(1)$ and $q \sim O(1)$, where order refers to order in the inverse aspect ratio expansion. Thus we choose the following notation:

$$
\bar{z}=z / a, R=R_{0}(1+\epsilon \bar{x}), \epsilon=a / R_{0}, \bar{\psi}=\psi / \psi_{\max }, d \bar{l}_{p}=d l_{p} / a,
$$

where $\psi_{\max }$ is the value of $\psi$ at the magnetic axis and we choose $\psi=0$ at the boundary. The scaled flux functions $\bar{p}(\bar{\psi})$, pressure, and $\bar{F}(\bar{\psi})$ are defined by

$$
\bar{F}=\frac{a^{2}}{R_{0} \psi_{\max }} F, \quad \bar{p}=\frac{\mu_{0} a^{4}}{\psi_{\max }^{2}} p .
$$

Henceforth, all equations in this paper will be expressed in scaled variables except where explicitly stated. We will omit the bars for convenience. In the asymptotic limit $\epsilon \rightarrow 0$, with $p, q \sim O(1)$, the solution of the Grad-Shafranov equation for $\psi(x, z)$ splits into two regions. These are the Core Region where $\psi=\psi(x)$ (i.e., $\psi$ is independent of $z$ ), and the Boundary Layer Region (with width of order $\epsilon^{1 / 2}$ ) adjacent to the conducting wall where $\nabla \psi$ is $O\left(\epsilon^{-1 / 2}\right)$ and normal to the wall. Typical flux surfaces (for $d p / d \psi>0$ ) are shown in Fig. 2. 
When $p^{\prime}>0$ the core solution, $\psi(x)$, is given implicitly by ${ }^{1}$

$$
2 \pi \int_{0}^{\psi_{0}} \frac{q\left(\tilde{\psi}_{0}\right)}{\sqrt{2\left(C-p\left(\tilde{\psi}_{0}\right)\right)}} d \tilde{\psi}_{0}=\int_{-1}^{x} \ell(\tilde{x}) d \tilde{x},
$$

where $\ell(x)$ is the vertical height of the vacuum vessel at $x$, see Fig. 1 . The constant $C$ is given, again implicitly, by

$$
\int_{0}^{1} \frac{q\left(\tilde{\psi}_{0}\right)}{\sqrt{C-p\left(\tilde{\psi}_{0}\right)}} d \tilde{\psi}_{0}=A_{0}
$$

where $A_{0}$ is the area of the poloidal cross section of the vacuum vessel. We will assume that $\beta$ is small enough that a solution to Eq. (5) exists. ${ }^{1}$ When a solution to Eq. (5) does not exist we have a new kind of solution that we termed "hole" solutions. These solutions are described in $C K^{-3}$. The core solution puts the magnetic axis at $x \sim 1-O\left(\epsilon^{1 / 2}\right)$ for $p^{\prime}>0$. When $p^{\prime}<0$ the Shafranov shift is negative and the magnetic axis is at $x \sim-1+O\left(\epsilon^{1 / 2}\right) .{ }^{1}$

The boundary layer solution yields ${ }^{1}$

$$
\frac{1}{2}\left(\frac{\partial \psi_{0}}{\partial s}\right)^{i}=\frac{2}{\epsilon} \int_{\psi_{\text {core }}(x)}^{\psi_{0}} d \psi^{\prime}\left(X\left(\psi^{\prime}\right)-x\right) \frac{d p}{d \psi^{\prime}}
$$

where $s$ is the perpendicular distance from the conducting wall. The function $X_{0}\left(\psi^{\prime}\right)$ in Eq. (6) is the $x$ position of the $\psi^{\prime}$ surface in the core and $\psi_{\text {core }}(x)$ is the core solution [i.e., a solution of Eq. (4)]. The boundary layer solution matches the core solution when $s\left(p^{\prime} / \epsilon\right)^{1 / 2} \gg 1$. The equilibrium solution is valid when $\epsilon \ll 1$ and when the boundary layer is narrow compared to the minor radius; that is when $\beta \gg t / q^{2}$. One can integrate Eq. (6) to obtain $s=s(\psi, x) .{ }^{1}$ This is not, however, necessary for the following analysis: from Eqs. (6) and (3) we find the poloidal field, $B_{p}$, in the boundary layer to be of order $\sqrt{\epsilon p}$. The ratio of poloidal field strength in the boundary layer to poloidal field strength in the core is roughly $\left(\epsilon / q^{2} \beta\right)^{\frac{1}{2}}$.

The magnetic field strength is important for our transport calculations. A simple calculation yields,

$$
B^{2}=\left[\frac{F^{2}(0)}{R_{0}^{2}}-2 \mu_{0} p(\psi)\right]-2 \epsilon x \frac{F^{2}(0)}{R_{0}^{2}}+4 \mu_{0} \epsilon \int_{-1}^{x} d x^{\prime} p\left(x^{\prime}\right)
$$

where $p(x)=P\left(\psi_{\text {core }}(x)\right)$ is the pressure profile in the core (in unnormalized units) and $F(0)=R_{0} \psi_{\max } \sqrt{2 C} / a^{2}$ is the toroidal flux function at the wall. There are two important features of $B^{2}$. First, $B^{2}$ is a constant on the flux surface in the core. Second, the variation of $B^{2}$ on a surface is $\mathcal{O}(\epsilon)$ but the variation across the surfaces is $\mathcal{O}(\beta)$. Thus surfaces of constant $B^{2}$ are closed for $\beta \gg \epsilon$ and they almost coincide with flux surfaces. 


\section{Guiding Center Motion and Heuristic Estimates of Neoclassical Transport}

In this section, we consider guiding center motion in high $\beta$ large aspect ratio equilibrium. We shall not consider strongly rotating tokamaks - therefore, the equilibrium electric fields are weak. The guiding center motion in the poloidal plane can be deduced from three approximately conserved quantities, $E=(1 / 2) m v^{2}$ the kinetic energy, $\mu=(1 / 2) m v_{\perp}^{2} / B$ the magnetic moment and, $\bar{p}_{\phi}=(e / c) \psi-m v_{\|} R$ the gyrophase averaged toroidal canonical angular momentum. The notation $v_{\perp}, v_{\|}$means respectively the velocity perpendicular to the magnetic field and the velocity parallel to the magnetic field. We drop species labels in this heuristic discussion because electrons and ions behave similarly. Clearly,

$$
v_{\|}=\sqrt{\frac{2}{m}(E-\mu B)} .
$$

Substituting this value of $v_{\|}$into the equation $\bar{p}_{\phi}=$ constant yi $\leqslant$ Ids an equation describing the projection of the guiding center motion on the poloidal plane. These trajectories are parameterized by the values of the three constants, $\bar{p}_{\phi}, \mu$ and $\mathrm{E}$. In tokamaks of interest $\delta_{p}=c m v_{\|} R /(e \psi) \ll 1$. In this limit the particles stay close to a given flux surface. The variation of $B=|\mathbf{B}|$ on a surface is a factor of order $\epsilon$ [see Eq. (7)]. Note that $B$ does not vary on the straight surfaces in the core region. On a given surface $B$ takes its maximum value in the core region. Trapped particle turning points are located at $E=\mu B\left(\psi, x_{t}\right)$; clearly since $B$ is constant on the surface in the core region there are no turning points and no trapped particles in the core region. Let us define $B_{c}=B(\psi, X(\psi))$, i.e., $B_{c}$ is the value of $B$ in the core region, and let $\hat{B}=B / B_{c}$. It is convenient to define $\lambda$ so that $\lambda \hat{B}=\mu B / E$; clearly $\lambda$ is conserved in the guiding center motion. Thus,

$$
\frac{v_{\|}}{v}=\sigma(\sqrt{2(1-\lambda \hat{B})}) \text {, }
$$

where $\sigma$ takes the values plus or minus one.

Our definition of $\lambda$ is slightly different from the conventional definition. ${ }^{3}$ At a specific position on a flux surface, $\lambda$ takes the values $1<\lambda<1 / \hat{B}$ for trapped particles and $0<\lambda<1$ for passing particles. Thus a typical trapped particle has $v_{\|} / v \sim \mathcal{O}\left(\epsilon^{1 / 2}\right)$, like the low $\beta$ case. The fraction of trapped particles at a specific point on the flux surface, $f_{t}$, is (to lowest order in $\left.\delta_{p}\right) f_{t}=(1-\hat{B})^{1 / 2} \sim \mathcal{O}\left(\epsilon^{1 / 2}\right)$. We have assumed (correctly) that the distribution function is isotropic (i.e., independent of $\lambda$ ) to lowest order in $\delta_{p}$. Note that in the core $\hat{B}=1$ and as we have previously stated $f_{t}=0$. Let us find the fraction of trapped particles, $\left\langle f_{t}\right\rangle$ in the volume between the surfaces $\psi$ and $\psi+d \psi$. We shall further assume (again correctly) that to lowest order in $\delta_{p}$ the distribution function is independent of the position on the flux surface. Thus,

$$
\left\langle f_{t}\right\rangle=\frac{\oint \frac{d \ell_{p}}{B_{p}}(1-\hat{B})^{\frac{1}{2}}}{\oint \frac{d \ell_{p}}{B_{p}}} \simeq \mathcal{O}\left(\epsilon^{\frac{1}{2}}\left(\frac{\epsilon}{q \beta}\right)^{\frac{1}{2}}\right) .
$$


Since there are no trapped particles in the core, the total fraction of trapped particles on a flux surface is reduced from the low $\beta$ result $\left(\mathcal{O}\left(\epsilon^{\frac{1}{2}}\right)\right)$ by the fraction of the volume, approximately $\left(\epsilon / q^{2} \beta\right)^{\frac{1}{2}}$, that is in the boundary layer. Another way of understanding this result is to consider the field line starting at the outermost (largest $X$ ) point on the flux surface and going once around the torus in the poloidal direction back to the outermost point. In Fig. (3), we plot $\frac{v \|}{v}$ versus $\ell$ the distance along the field line for various $\lambda$. The poloidal field in the boundary layer is large and the distance along the field line from the outermost point to the core, $\ell_{B}$, is short, typically $\ell_{B} \sim \pi a / \sqrt{\epsilon \beta}$. The distance along the field line through the core region is $2 \pi R q$. Thus, a fraction $\left(\epsilon / q^{2}, \beta\right)^{\frac{1}{2}}$ of the field line length is in the boundary layer. In the boundary layer, the trapped particles are a fraction $\epsilon^{\frac{1}{2}}$ of the particles and in the core there are no trapped particles. Thus, the trapped particles are a fraction $\epsilon^{\frac{1}{2}}\left(\epsilon / q^{2} \beta\right)^{\frac{1}{2}}$ of the total number of particles on a given field line. It is also clear that a passing particle spends roughly a fraction $\left(\epsilon / q^{2} \beta\right)^{\frac{1}{2}}$ of its time in the boundary layer. We have belabored the preceding point because it is the reduction of the trapped particle fraction (and the time a passing particle spends in the boundary layer) that gives rise to the high $\beta$ neoclassical transport coefficients being reduced from their (nominal) low $\beta$ values.

Let us now consider the banana widths of trapped particles. The banana width is defined at the outermost point on the flux surface. Let $\Delta \psi$ be the change in $\psi$ across the banana width. From $\bar{p}_{\phi}$ conservation we obtain

$$
\Delta \psi=\frac{2 c m v_{\| \max } R}{e}
$$

where $v_{\| \max }$ is the $v_{\|}$at the outermost point on the flux surface. Note that the number of flux surfaces in a banana width is independent of the spacing of the flux surfaces. The actual banana width, $\Delta_{B}$, is

$$
\Delta_{B}=\frac{v_{\| \max }}{v_{t h}} \rho_{p} \sim \mathcal{O}\left(\frac{\rho_{t}}{\sqrt{\beta}}\right),
$$

where $\rho_{p}$ is the poloidal larmor radius at the outermost point and $\rho_{t}$ is the toroidal larmor radius. The estimate in Eq. (12) is obtained by taking $v_{\| \max } \sim \epsilon^{1 / 2} v_{t h}$ and $B_{p} \sim \sqrt{\epsilon p}$. The banana width, $\Delta_{B}$, is reduced from its low $\beta$ value because of the increased poloidal field in the boundary layer. Note (again) that the number of banana widths in the boundary layer width is independent of the pressure. It is convenient to label flux surfaces by their $x$ position in the core $X(\psi)$. The poloidal field in the core is $B_{p o}(\psi)=\frac{1}{R_{o} a} \frac{d \psi}{d X}$ and the core poloidal larmor radius is $\rho_{p o}=\frac{v_{\text {sh } m c}}{e B_{p o}}$. Thus, the banana width in $\mathrm{X}, \Delta X_{B}$, is

$$
\Delta X_{B}=\frac{v_{\| B}}{v_{t h}} \frac{\rho_{p o}}{a} \sim \mathcal{O}\left(\epsilon^{\frac{1}{2}} \frac{\rho_{p o}}{a}\right)
$$

Let us now consider time scales for the particle motion. First we consider the bounce 
frequency, $\omega_{B}$, of a trapped particle.

$$
\frac{1}{\omega_{B}}=\oint \frac{d \ell}{v_{\|}}=\frac{1}{v} \oint \frac{B}{B_{p}} \frac{d \ell_{p}}{(1-\lambda \hat{B})^{\frac{1}{2}}} \sim \mathcal{O}\left(\frac{1}{\sqrt{\beta}} \frac{R}{v_{t h}}\right),
$$

where we have given the typical size of $\omega_{B}$. Similarly, the transit frequency, $\omega_{T}$, of a passing particle is

$$
\begin{aligned}
\frac{1}{\omega_{T}} & =\frac{1}{v} \oint \frac{B}{B_{P}} \frac{d \ell_{p}}{(1-\lambda \hat{B})^{\frac{1}{2}}} \\
& \sim \mathcal{O}\left(\frac{2 \pi q R}{v_{t h}}\right), v_{\|} \sim v_{t h} \\
& \sim \mathcal{O}\left(\frac{2 \pi q R}{v_{t h} \epsilon^{\frac{1}{2}}}\right), v_{\|} \sim \epsilon^{\frac{1}{2}} v_{t h} .
\end{aligned}
$$

The first estimate of $\omega_{T}$ is for for deeply passing particles and the second for barely passing particles. We note that for $q^{2} \beta \sim \mathcal{O}(1), \omega_{B} \sim \mathcal{O}\left(\omega_{T}\right)$ for deeply passing particles. We may distinguish four collisionality regimes. First the collisionless "banana" regime:

$$
\nu_{e f f}=\frac{\nu}{\epsilon} \ll \frac{\epsilon^{\frac{1}{2}} v_{t h}}{2 \pi q R}
$$

where $\nu$ is the collision rate and $\nu_{\text {eff }}$ is the effective collision rate for pitch angle scattering of a particle from the trapped to the passing regions of velocity space. We shall concentrate on this regime because it is appropriate for hot reactor like plasmas. The second regime, an intermediate collisionality regime where the barely passing particles are collisional is deined by,

$$
\frac{v_{t h}}{2 \pi q R} \gg \frac{\nu}{\epsilon} \gg \frac{\epsilon^{\frac{1}{2}} v_{t h}}{2 \pi q R}
$$

In this regime, both the banana particles and the deeply passing particles are collisionless, and barely passing particles are collisional. This regime is peculiar to high $\beta$ tokamaks. The third regime is the plateau regime where,

$$
\frac{v_{t h}}{2 \pi q R} \gg \nu \gg \frac{\epsilon v_{t h}}{2 \pi q R} \text {. }
$$

In this regime the trapped and barely passing particles are collisional and the deeply passing particles are collisionless. The third regime is the fully collisional regime, where all particles are collisional and

$$
\nu \gg \frac{v_{t h}}{2 \pi q R}
$$


Neoclassical transport (as distinguished from classical transport) arises from the guiding center drifts off the $\psi$ surfaces. Particles drift relative to the flux surface in the boundary layer but not in the core. Hence, neoclassical transport essentially takes place in the boundary layer. This does not mean that the core has no role; in fact, collisions on passing particles in the core relax distortions of the (passing) distribution function (including the resistive relaxation of the parallel current).

Let us estimate a typical banana regime perpendicular transport coefficient. To avoid complications of momentum conservation we consider the thermal diffusivity $\chi$. This diffusivity is expressed $i_{1} X$ space. The transport is a random walk of trapped particles with step length $\Delta X_{B}$ and frequency $\nu_{e f f}$ (the effective collision rate). The thermal diffusivity is weighted by the fraction of trapped particles on the flux surface. Thus,

$$
\chi \sim\left\langle f_{t}\right\rangle \nu_{e f f}\left(\Delta X_{B}\right)^{2} \sim \mathcal{O}\left(\left(\frac{\epsilon}{q^{2} \beta}\right)^{\frac{1}{2}} \epsilon^{\frac{1}{2}} \nu \frac{\rho_{p o}^{2}}{a^{2}}\right) .
$$

The reduction of $\chi$ from the low $\beta$ result by a factor $\left(\epsilon / q^{2} \beta\right)^{\frac{1}{2}}$ is due to the reduction in the trapped particle fraction. We note that the barely passing particle's contribution to $\chi$ is similar to $\mathrm{Eq}_{\mathrm{q}}$ (20). For barely passing particles their contribution to the diffusivity must be weighted by the fraction of time they spend in the boundary layer (approximately $\left(\epsilon / q^{2} \beta\right)^{1 / 2}$ - si $i_{,+}$ce only in the boundary layer do they drift off the $\psi$ surface. Quantitative calculations in the next section confirm the form of the estimate, Eq. (20), for the thermal diffusivity. The bootstrap current is also reduced from its low $\beta$ value by the factor $\left(\epsilon / q^{2} \beta\right)^{1 / 2}$ because it is driven by the deviations from the flux surface (in the usual manner ${ }^{3}$ ) in the boundary layer and damped by friction in the core. In all collisionality regimes, the neoclassical coefficients will be reduced by the factor $\left(\epsilon / q^{2} \beta\right)^{\frac{1}{2}}$, the ratio of the boundary layer volume to the core volume. We note that the classical transport coefficients are enhanced by a factor $\left(q^{2} \beta / \epsilon\right)^{\frac{1}{2}}$, but they are still negligible compared to the neoclassical coefficients in the banana regime.

We shall give the banana regime neoclassical coefficients in the next section. The other regimes of collisionality are not hard to calculate but they are not thought to be relevant.

\section{Formal Neoclassical Theory of the Banana Regime}

The general formalism of neoclassical theory is well developed and we shall not try to describe it here. We shall follow the rotation of Hinton and Hazeltine's review article. ${ }^{3}$ The approach we adopt was first used by Rosenbluth, Hazeltine and Hinton ${ }^{4}$ to obtain the transport coefficients (the Onsager Matrix) in a large-aspect ratio low $\beta$ tokamak. When the variation of $|\mathbf{B}|$ over a surface is small, the fraction of trapped particles (at any point) is small and well localized in pitch angle. In this case, pitch angle scattering of trapped and barely passing particles dominates the collisions. Rosenbluth, et al., used a 
variational principle for the transport coefficients in the banana regime. This variational principle is directly applicable to the high $\beta$ case; the only difference is the evaluation of the flux surface average of the pitch angle integrals. Glasser and Thompson ${ }^{5}$ have stressed the validity of the Rosenbluth, Hazeltine and Hinton variational principle in shaped tokamaks with a small trapped particle fraction. As in the previous section, we use the flux surface position $\mathrm{X}(\psi)$ in the core to label the surfaces and we normalize $|\mathrm{B}|$ to its core value, i.e., $\hat{B}=B / B_{c}$. Let us define the electron collision time, $\tau_{e}$, the ion collision time $\tau_{i}$ and the parallel conductivity, $\sigma_{\|}$, as: ${ }^{3}$

$$
\begin{aligned}
\frac{1}{\tau_{e}} & =\frac{4}{3}(2 \pi)^{\frac{1}{2}}\left[\frac{n_{e} Z_{i} e^{4} \ln \Lambda}{m_{e}^{\frac{1}{2}} T_{e}^{\frac{3}{2}}}\right], \\
\frac{1}{\tau_{i}} & =\frac{4}{3} \pi^{\frac{1}{2}} \frac{n_{i} Z_{i}^{4} e^{4} \ell n \Lambda}{m_{i}^{\frac{1}{2}} T_{i}^{\frac{3}{2}}} \\
\sigma_{\|} & \approx \frac{n_{e} e^{2} \tau_{e}}{m_{e}}\left[0.29+\frac{0.46}{1.08+Z_{i}}\right]^{-1} .
\end{aligned}
$$

The coulomb logarithm, $\ell n \Lambda$, is typically $17-20$. We shall also use the notation

$$
\langle A\rangle=\frac{\oint \frac{d \ell_{p}}{B_{p}} A}{\oint \frac{d \ell_{p}}{B_{p}}} .
$$

The electron particle flux through the $\mathrm{X}$ :urface, $\Gamma_{e}$, is,

$$
\begin{gathered}
\Gamma_{e}=-I_{11} \frac{n_{e} \rho_{\text {epo }}^{2}}{\tau_{e} a}\left[\left(\frac{1}{p_{e}} \frac{d p_{e}}{d X}+\frac{T_{i}}{Z_{i} T_{e} p_{i}} \frac{d p_{i}}{d X}-\frac{1.2}{Z_{i} T_{e}} \frac{d T_{i}}{d X}\right)\left(0.35+0.18 / Z_{i}\right)\right. \\
\left.-\frac{1}{T_{e}} \frac{d T_{e}}{d X}\left(0.5+0.2 / Z_{i}\right)\right]-I_{11} \frac{n_{e} c}{B_{p o}} \frac{\left\langle E_{\|} \hat{B}\right\rangle}{\left\langle\hat{B}^{2}\right\rangle}\left(0.73+0.49 / Z_{i}\right),
\end{gathered}
$$

where $\rho_{\text {epo }}=\frac{v_{\text {sele }} m_{\theta} c}{e B_{p o}} . I_{11}$ is

$$
\begin{aligned}
I_{11} & =\left\langle(1-\hat{B})^{\frac{1}{2}}\left[\ln \left(\frac{2(1-\hat{B})^{\frac{1}{2}}}{\left\langle f_{t}\right\rangle}\right)-1\right]\right\rangle \\
& \sim \mathcal{O}\left(\epsilon^{\frac{1}{2}}\left(\frac{\epsilon}{q^{2} \beta}\right)^{\frac{1}{2}} \ln \epsilon^{-\frac{1}{2}}\right)
\end{aligned}
$$

(see Appendix A for the reduction from $I_{11}$ given in Hinton and Hazeltine) where $\left\langle f_{t}\right\rangle=$ $\left\langle(1-\hat{B})^{\frac{1}{2}}\right\rangle$ is the fraction of trapped particles on the surface [see Eq. (10)]. The electron 
heat flux, $q_{e}$ is

$$
\begin{gathered}
\frac{q_{e}}{T_{e}}=-I_{11} \frac{n_{e} \rho_{e p o}^{2}}{\tau_{e} a}\left[-\left(\frac{1}{p_{e}} \frac{d p_{e}}{d X}+\frac{T_{i}}{Z_{i} T_{e} p_{i}} \frac{d p_{i}}{d X}-\frac{1.2}{Z_{i} T_{e}} \frac{d T_{i}}{d X}\right)\left(0.5+0.2 / Z_{i}\right)\right. \\
\left.+\frac{1}{T_{e}} \frac{d T_{e}}{d X}\left(1.2+0.47 / Z_{i}\right)\right]+I_{11} \frac{n_{e} c}{B_{p o}} \frac{\left\langle E_{\|} \hat{B}\right\rangle}{\left\langle\hat{B}^{2}\right\rangle}\left(0.9 / Z_{i}\right)
\end{gathered}
$$

The ion heat flux, $q_{i}$, is

$$
\frac{q_{i}}{\Gamma_{i}}=-0.33 I_{11} \frac{n_{i} \rho_{i p o}^{2}}{\tau_{i} a}\left[-\frac{1}{T_{i}} \frac{d T_{i}}{d X}\right]
$$

where $\rho_{i p o}=\frac{v_{\text {thi }} m_{i} c}{Z e B_{\text {po }}}$. Finally (perhaps the only relevant equation) the "flux of flux" equation, i.e., the current equation, is

$$
\begin{gathered}
\left\langle J_{\|} \hat{B}\right\rangle=-I_{11} \frac{n_{e} T_{e} c}{a B_{p o}}\left[\left(\frac{1}{p_{e}} \frac{d_{\tilde{r}}}{d x} \therefore \frac{T_{i}}{N_{e} p_{i}} \frac{d p_{i}}{d X}-\frac{1.2}{Z_{i} T_{i}} \frac{d T_{e}}{d X}\right)\left(0.75+0.5 / Z_{i}\right)+\frac{0.9}{T_{e}} \frac{d T_{e}}{d X}\right] \\
+\left[1-\left(0.75+0.27 / Z_{i}\right) I_{11}\right] \sigma_{\|} \frac{\left\langle E_{\|} \hat{B}\right\rangle}{\left\langle\hat{B}^{2}\right\rangle} .
\end{gathered}
$$

This equation determines $\left\langle E_{\|} \hat{B}\right\rangle$ since, from Ampere's law,

$$
\left\langle J_{\|} \hat{B}\right\rangle=\frac{c F(X)}{a 4 \pi \ell(X)} \frac{d}{d X}\left[\frac{1}{F} \oint d \ell_{p} B_{p}\right] .
$$

The first term on the right hand side of Eq. (27) is the bootstrap current. It is common to replace $\left\langle E_{\|} \hat{B}\right\rangle$ using

$$
\left(\frac{\partial \psi}{\partial t}\right)_{\phi}=\frac{B_{c}(X)}{F(X)} \frac{c\left\langle E_{\|} \hat{B}\right\rangle}{\left\langle R^{-2}\right\rangle} \simeq c R_{0}\left\langle E_{\|} \hat{B}\right\rangle
$$

As we have predicted all the neoclassical coefficients in Engs. (23), (25), (26) and (27) are reduced by the factor $\left(\epsilon / q^{2} \beta\right)^{\frac{1}{2}}$. The neoclassical correction to the conductivity is negligible.

Let us estimate the size of the bootstrap current, $J_{b s}$, and the actual current, $J_{\|}$. From the equilibrium equations, Eqs. (4), (6), and (28) we find $J_{\|} \sim c \sqrt{\epsilon p} / a$ and from Eqs. (25) and (27) we find $J_{b s} \sim c \sqrt{p} / a$. Thus,

$$
\frac{J_{\| 1}}{J_{b s}} \sim \epsilon^{\frac{1}{2}}
$$

The electric field must, therefore, balance the bootstrap current term, not the current itself. In this case, the sign of the electric field is opposite to the current. Recall that at 
low $\beta$ the electric field is in the direction of the current. Thus, we have "Ohmic Cooling" in high $\beta$ plasmas - i.e., the plasma gives energy to the transformer. The magnitude of the ohmic cooling is small in fact it is a factor $\epsilon\left(\epsilon / q^{2} \beta\right)^{\frac{1}{2}}$ smaller than the electron neoclassical thermal conduction (which is itself usually dwarfed by anomalous transport). At the magnetic axis the bootstrap current is zero (since the pressure gradient vanishes on axis) and the actual current balances the electric field. Thus, on axis the poloidal flux is dropping and off axis it is rising. The q profile therefore evolves towards a hollow profile. The evolution of the current is complicated - it involves a boundary layer near the magnetic axis where $J_{\|} \sim J_{b}$ and the equation is part of a messy integrodifferential system. Let us therefore leave its sclution for further study! We note that the claim in $\mathrm{CK}{ }^{3}$ that the loop voltage scales as $T^{-\frac{1}{2}}$ was incorrect and in fact it scales as $T^{-1}$ - an unfortunate proof reading error made in haste.

\section{Conclusion}

In this paper, we have considered neoclassical transport in high $\beta$ large aspect ratio tokamaks. The $|\mathbf{B}|$ surfaces are clos $2 d$ and they coincide with the flux surfaces in the core. In the boundary layer $|\mathbf{B}|$ varies by a factor $\epsilon$ (the inverse aspect ratio) on a llux surface. Equilibrium properties are summarized in Sec. II. Trapped particles are confined to the boundary layer and they are a fraction $\epsilon^{\frac{1}{2}}$ of the particles in the boundary layer. However, the boundary layer is only a fraction $\left(\epsilon / q^{2} \beta\right)^{\frac{1}{2}}$ of the total volume. Thus, the fraction of ail particles that are trapped, $\left\langle f_{t}\right\rangle$, is roughly $\epsilon^{\frac{1}{2}}\left(\epsilon / q^{2} \beta\right)^{\frac{1}{2}}$. Guiding center motion and the fraction of trapped particles are discussed in Sec. III. Formal neoclassical theory (in Sec. IV) shows that all neoclassical coefficients are reduced from their low $\beta$ values by the factor $\left(\epsilon / q^{2} \beta\right)^{\frac{1}{2}}$. The transport coefficients are given in Eqs. (23), (25), (26) and (27). The evolution of the poloidal flux, Eq. (27), is dominated by the bootstrap current. The bootstrap current is a factor $\epsilon^{\frac{1}{2}}$ bigger than the actual parallel current (except at the magnetic axis). Sustaining a steady state high $\beta$ tokamak may be problematic because current must be driven on axis and suppressed off axis. This issue is beyond the scope of this paper and it is probably not amenable to analytical analysis.

Further studies of high $\beta$ large aspect ratio equilibria are in progress. Many issues remain to be investigated and it is difficult to predict which issues are the most critical. Clearly, kink mode stability is an issue since the current gradient in the boundary layer is large. High $\beta$ will only be attractive if anomalous transport improves at high $\beta$. Unfortunately, we don't really understand ti ansport at low $\beta$. Thus, predicting transport at high $\beta$ is at best speculative. Howeve: , the favorable properties that ensure the ballooning stability (large local shear and si ort connection length in the bad curvature region) may also be beneficial for transport. These issues are being pursued.

Acknowledgments. I wish to thank Russell Kulsrud and Ernie Valeo for useful discussions. This work was supported by the United States Department of Energy Contract No. DE-ACO2-76-CHO-3073. 


\section{Appendix: Evaluation of the Flux Surface and Pitch Angle Integrals}

The variational treatment of banana regime transport in a large aspect ratio tokamak, first introduced by Rosenbluth, Hazeltine and Hinton, ${ }^{4}$ is easily generalized to all axisymmetric equilibria with a small fraction of trapped particles at every point on the surface. ${ }^{5}$ We follow the notation of Hinton and Hazeltine ${ }^{3}$ (Sec. VI.B). The transport coefficients are given in terms of the following integrals:

$$
\begin{aligned}
& I_{11}=\left\langle\int_{1}^{1 / \dot{B}} \frac{d \lambda \lambda}{(1-\lambda \hat{B})^{1 / 2}}+\int_{0}^{1} d \lambda \lambda\left[\frac{1}{(1-\lambda \hat{B})^{1 / 2}}-\frac{1}{\left\langle(1-\lambda \hat{B})^{1 / 2}\right\rangle}\right] /\right. \\
& I_{13}=\left\langle\int_{1}^{1 / \dot{B}} \frac{d \lambda \lambda \hat{B}^{2}}{(1-\lambda \hat{B})^{1 / 2}}+\int_{0}^{1} d \lambda \lambda\left[\frac{\hat{B}^{2}}{(1-\lambda \hat{B})^{1 / 2}}-\frac{\left\langle\hat{B}^{2}\right\rangle}{\left\langle(1-\lambda \hat{B})^{1 / 2}\right\rangle}\right]\right\rangle
\end{aligned}
$$

and

$$
I_{33}=\left\langle\int_{1}^{1 / \dot{B}} \frac{d \lambda \lambda \hat{B}^{4}}{(1-\lambda \hat{B})^{1 / 2}}+\int_{0}^{1} d \lambda \lambda\left[\frac{\hat{B}^{4}}{(1-\lambda \hat{B})^{1 / 2}}-\frac{\left\langle\hat{B}^{4}\right\rangle}{\left\langle(1-\lambda \hat{B})^{1 / 2}\right\rangle}\right]\right\rangle .
$$

The angle brackets are defined in Eq. (22). In labeling orders we shall treat $\epsilon /\left(q^{2} \beta\right) \sim$ $\mathcal{O}(\epsilon)$. Let us consider first $I_{11}$. We may rewrite $I_{11}$ as

$$
I_{11}=\left\langle\frac{4}{3}\left(\frac{1}{\hat{B}^{2}}-1\right)\right\rangle+\int_{0}^{1} \frac{d \lambda \lambda f(\lambda)}{\left[(1-\lambda)^{1 / 2}+f(\lambda)\right](1-\lambda)^{1 / 2}}
$$

where

$$
f(\lambda)=\left\langle(1-\lambda \hat{B})^{1 / 2}-(1-\lambda)^{1 / 2}\right\rangle .
$$

Note that $\hat{B}=1$ except in the boundary layer where $B_{p} / B_{p o} \sim \mathcal{O}(\epsilon)$. Thus, $\left\langle\hat{B}^{-2}-1\right\rangle \sim$ $\mathcal{O}\left(\epsilon^{3 / 2}\right)$. We shall ignore $\mathcal{O}\left(\epsilon^{3 / 2}\right)$ terms since our final answer is $\mathcal{O}(\epsilon)$. When $(1-\lambda) \sim \mathcal{O}(\epsilon)$, then $f(\lambda) \sim \mathcal{O}(\epsilon)$ and when $(1-\lambda) \gg \mathcal{O}(\epsilon)$, then $f(\lambda) \sim \mathcal{O}\left(\epsilon^{3 / 2}\right)$. Therefore, $(1-\lambda)^{1 / 2} \gg$ $f(\lambda)$ except where $(1-\lambda) \sim \mathcal{O}\left(\epsilon^{2}\right)$. If $(1-\lambda) \sim \mathcal{O}\left(\epsilon^{2}\right)$, then $f(\lambda)=f(1)+\mathcal{O}\left(\epsilon^{3 / 2}\right)$. And we note from Eqs. (10) and (35) that $f(1)=\left\langle f_{t}\right\rangle$. thus in the integral in the second term on the right hand side of Eq. (34) we may set $\left[(1-\lambda)^{1 / 2}+f(\lambda)\right] \sim\left[(1-\lambda)^{1 / 2}+\left\langle f_{t}\right\rangle\right]$. Thus,

$$
I_{11}=\left\langle\int_{0}^{1} \frac{d \lambda \lambda}{\left[(1-\lambda)^{1 / 2}+\left\langle f_{t}\right\rangle\right]}\left(\frac{(1-\lambda \hat{B})^{1 / 2}}{(1-\lambda)^{1 / 2}}-1\right)\right\rangle+\mathcal{O}\left(\epsilon^{3 / 2}\right) .
$$

The $\lambda$ integral can be done asymptotically to $\mathcal{O}(\epsilon)$. We obtain,

$$
I_{11}=\left\langle(1-\hat{B})^{1 / 2}\left[\ln \left(\frac{2(1-\hat{B})^{1 / 2}}{\left\langle f_{t}\right\rangle}\right)-1\right]\right\rangle+\mathcal{O}\left(\epsilon^{3 / 2}\right) .
$$

It is trivial to show that $I_{13} \sim I_{11}+\mathcal{O}\left(\epsilon^{3 / 2}\right)$ and that $I_{33} \sim I_{11}+\mathcal{O}\left(\epsilon^{3 / 2}\right)$. The logarithmic enhancement comes from the collisions on the barely passing particles. 


\section{References}

'S.C. Cowley, P.K. Kaw, R.S. Kelly, and R.M. Kulsrud, Phys. Fluids B, 3, 2069(1991).

${ }^{2}$ S.C. Cowley, Phys. Fluids B,3, 3357(1991).

${ }^{3}$ F.L. Hinton, and R.D. Hazeltine, Rev. Mod. Phys. 48, 239(1976).

${ }^{4}$ M.N. Rosenbluth, F.L. Hinton, and R.D. Hazeltine, Phys. Fluids, 15, 116(1972).

${ }^{5}$ A.H. Glasser, and W.B. Thompson, Phys. Fluids, 16, 95(1973). 


\section{Figures}

FIG. 1. Geometry of the equilibrium for an unusual boundary shape.

FIG. 2. Equilibrium flux surface illustrating the core region and the boundary layer region.

FIG. 3. The parallel velocity divided by the total velocity, $v_{\|} / v$, as a function of the length along the field line, $l$. Thie contours are different values of $\lambda$. 
PPPL $\$ 1 \times 0500$

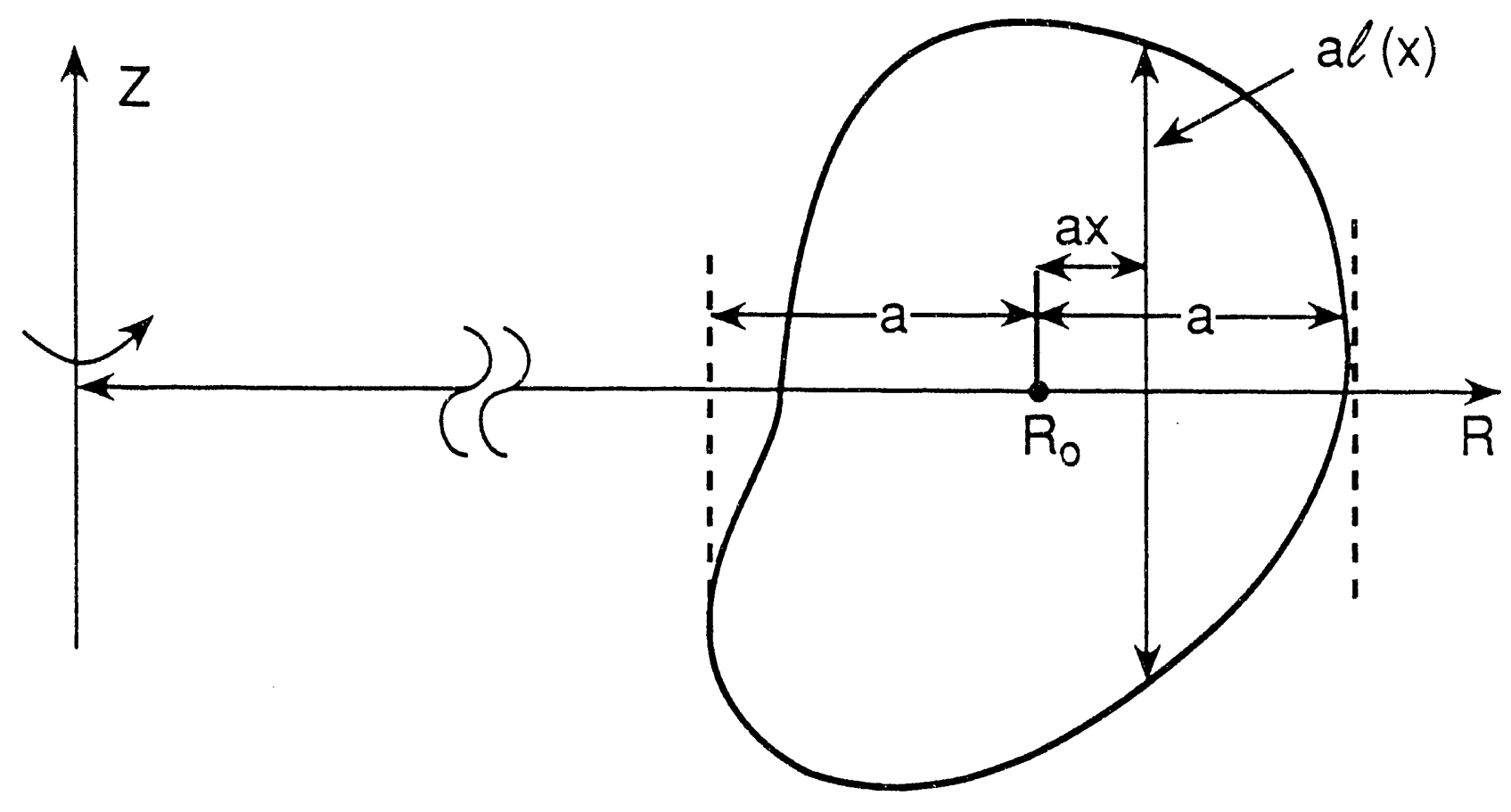

FIG. 1 
PPPL\#91X0301

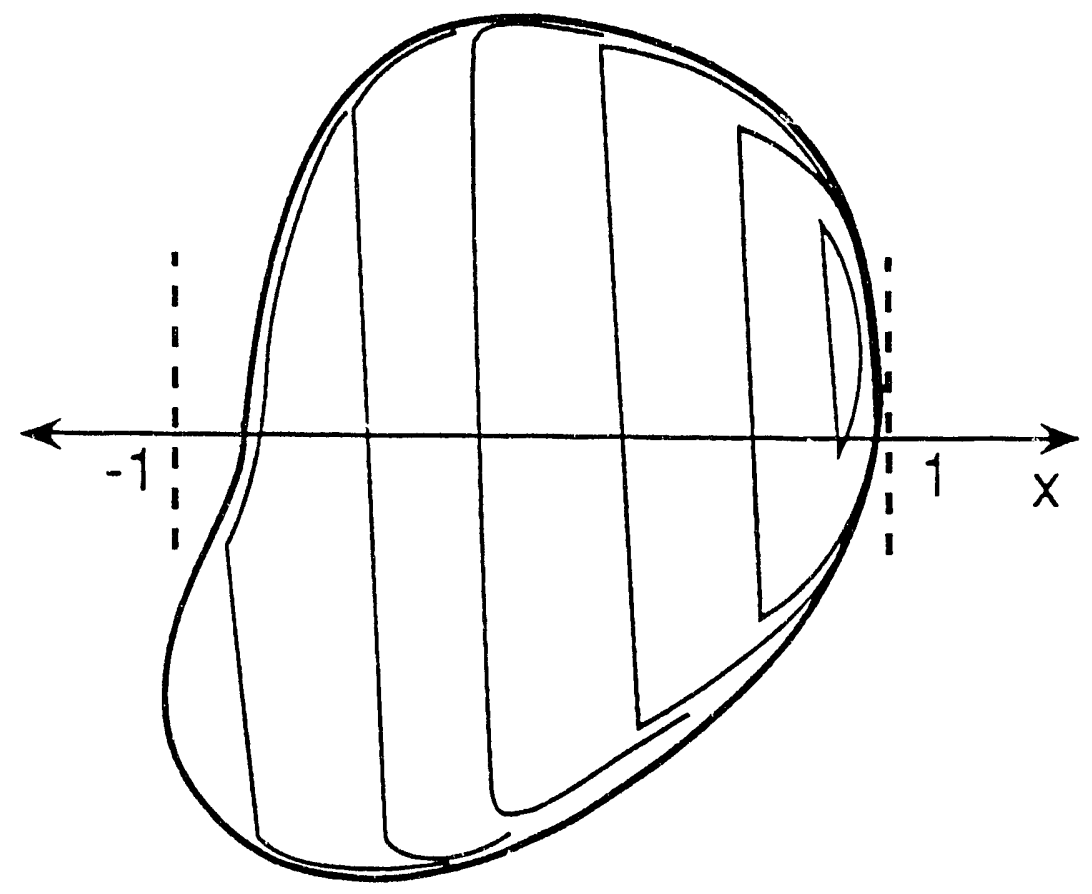

c

FIG. 2 


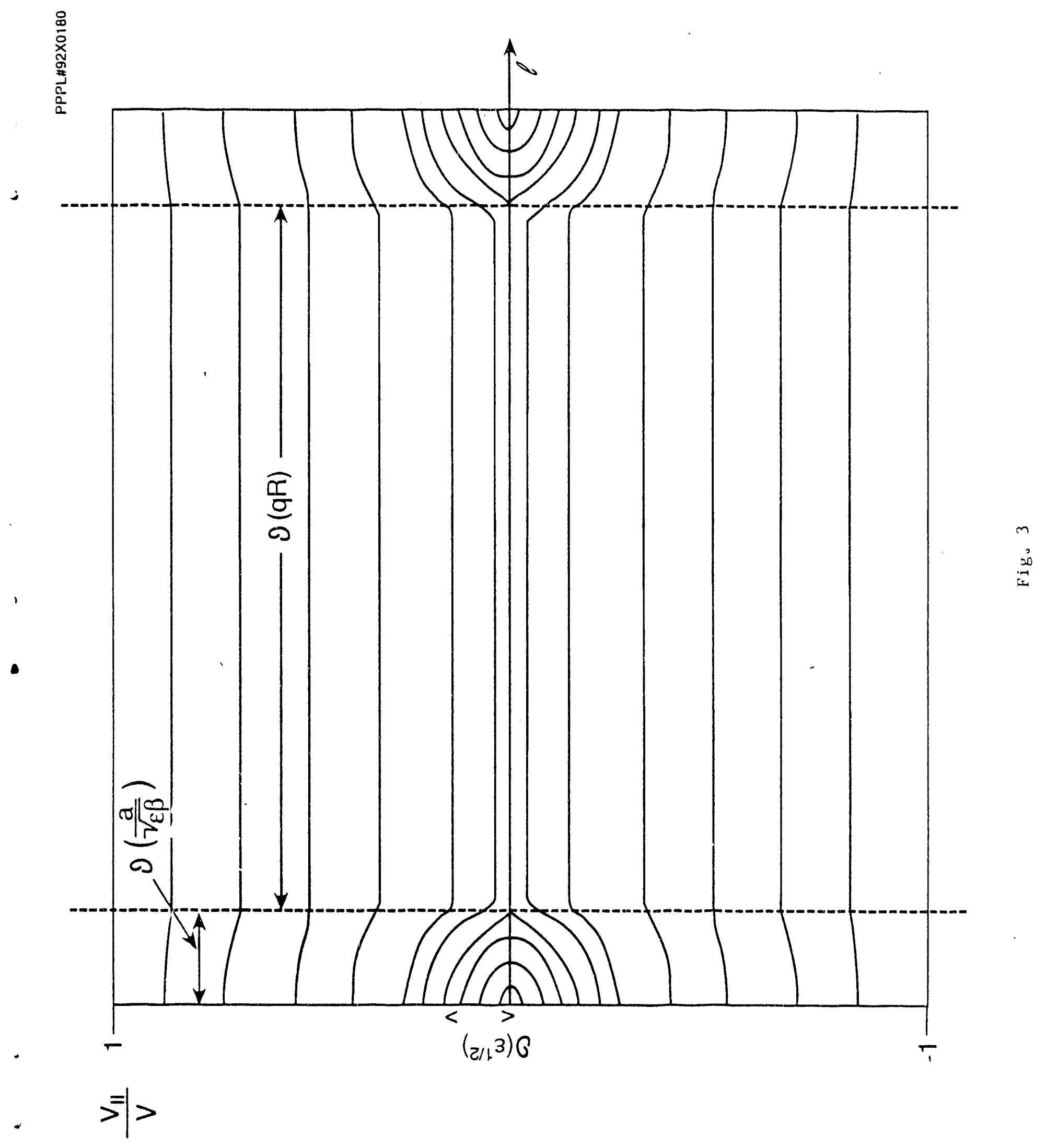


Dr. F. Paoloni, Univ. of Wollongong, AUSTRALIA

Frof. M.H. Brennan, Univ. of Sydnoy, AUSTRALIA

Plasma Research Lab., Australian Nat. Univ., AUSTRALIA

Prof. I.R. Jones, Flinders Univ, AUSTRALIA

Prof. F. Cap, Inst. for Theoretical Physics, AUSTRIA

Prot. M. Heindler, institut fur Theoretische Physik, AUSTRIA

Prof. M. Goossens, Astronomisch Instiwut, BELGIUM

Ecole Royale Militaire, Lab. de Phy. Plasmas, BELGIUM

Commission-Europsen, DG. XII-Fusion Prog., BELGIUM

Prot. R. Bouciqué, Rijksuniversiteit Gent, BELGIUM

Dr. P.H. Sakanaka, Instituto Fisica, BRAZIL

Instituto Naciona De Pesquisas Espaciais-INPE, BRAZIL

Documents Office, Atomic Energy of Canada LId., CANADA

Dr. M.P. Bachynskj, MPB Technologies, Inc., CANADA

Dr. H.M. Skarsgard, Univ. of Saskatchewan, CANADA

Prof. J. Teichmann, Univ. of Montreal, CANADA

Prot. S.R. Sroenivasen, Univ. of Calgary, CANADA

Prot. T.W. Johnston, INAS-Energi, CANADA

Dr. R. Botton, Centre canadien de tusion magnétique, CANADA

Dr. C.R. James,. Univ. of Aberta, CANADA

Dr. P. Lukác, Komenského Universzita, CZECHO-SLOVAKIA

The Librarian, Culham Laboratory, ENGLAND

Library, R61, Ruthortord Apploton Laboratory. ENGLAND

Mrs. S.A. Hutchinson, JET Librery, ENGLAND

Dr. S.C. Shama, Univ. of South Pacific, FIJI ISLANDS

P. Mathonen, Univ. of Helsinki, FINLAND

Prof. M.N. Bussac, Ecole Polytoctinique, FRANCE

C. Moutrot, Lab. do Physique des Milieux lonises, FRANCE

J. Radat, CEN/CADARACHE - Bat 506, FRANCE

Prof. E. Economou, Univ. of Crete, GREECE

Ms. C. Pinni, Univ. of loannina, GREECE

Dr. T. Mud, Acaderny Bibliographic Ser., HONG KONG

Proprint Library, Hungarian Acadomy of Sei., HUNGARY

Dr. B. DasGupta, Saha inst of Nuctear Ptysics, INDIA

Dr. P. Kaw, Inst. for Plasma Rosearch, INDIA

Dr. P. Rosenau, I real inst of Technology, ISPAEL

Librarian, Intomational Center for Theo Physics, ITALY

Miss C. Do Palo, Associaziono EURATOM-ENEA, ITALY

Dr. G. Grosso, Istituto di Fisica del Plasma, ITALY

Prof. G. Rostangni, lestuto Gas lonizzati Del Cnr, ITALY

Dr. H. Yamato, Toshiba Ros \& Dovel Center, JAPAN
Prot. I. Kawakami, Hiroshima Univ., JAPAN

Prof. K. Nishikawa, Hiroshima Univ., JAPAN

Director, Japan Atomic Energy Research Inst, JAPAN

Prof. S. Itoh, Kyushu Univ., JAPAN

Research Into. Ctr., National Instit. for Fusion Science, JAPAN

Prof. S. Tanaka, Kyoto Univ., JAPAN

Library, Kyoto Univ., JAPAN

Prot. N. Inowe, Univ. of Tokyo, JAPAN

Secrotary, Plasma Section, Electrotechnical Lab., JAPAN

S. Mori, Tectnical Advisor, JAERI, JAPAN

Dr. O. Mitarai, Kumamoto Inst. of Technology, JAPAN

J. Hyeon-Sook, Kores Abomic Energy Research Inst. KOREA

D.I. Choi, The Korea Adv. Inst of Sai. \& Tech., KOREA

Prof. B.S. Liby, Univ. of Waikato, NEW ZEALAND

Inst of Physics, Chinese Acad Sci PEOPLE'S REP. OF CHINA

Library, Inst of Plasma Physics, PEOPLE'S REP. OF CHINA

Tsinghua Univ. Library, PEOPLE'S REPUBLIC OF CHINA

Z. L.S.W. Inet Physics, PEOPLE'S REPUBLIC OF CHINA

Prof. J.A.C. Cabra, Instituto Superior Tocnico, PORTUGAL

Dr. O. Petrus, ALI CUZA Univ., ROMANIA

Dr. J. de Villiers, Fusion Studies, AEC, S. AFRICA

Prof. M.A. Hellborg, Univ. of Natal, S. AFRICA

Prot. D.E. Kim, Pahang Inst. of Sai. \& Tech., SO. KOREA

Prof. C.I.E.M.A.T, Fusion Division Library, SPAIN

Dr. L. Stontio, Univ. of UMEA, SWEDEN

Librey, Royal Inst. of Tecthology, SWEDEN

Prot. H. Witholmson, Chaimers Univ. of Tech., SWEDEN Centre Phys. Des Plasmas, Ecole Polytech, SWITZERLAND Bibliothoek, Inst. Voor Plasma-Fysica, THE NETHERLANDS Asst Prot. Dr. S. Celkir, Nidds East Tech. Univ., TURKEY Dr. V.A. Gukhikh,Sci. Res. Inst Electrophys.I Apparatus, USSR

Dr. D.D. Ryubv, Siberian Branch of Academy of Sai., USSR

Dr. G.A. Eliseov, I.V. Kurchatov Inst, USSR

Librarien, The Ukr.SSR Academy of Sciences, USSR

Dr. LM. Kowizhnykh, Inst. of Gonoral Physics, USSR

Kerniorsctungsaniage GmbH, Zentralbibliathek, W. GERMANY

Bibliothek, Inst. For Plasmatorschung, W. GERMANY

Prof. K. Schindler, Ruhr-Universitat Bochum, W. GERMANY

Dr. F. Wagner, (ASDEX), Max-Planck-Institut, W. GERMANY

Librarian, Max-Plenck-Institut, W. GERMANY

Prof. R.K. Janev, Inst of Ptysics, YUGOSLAVIA 

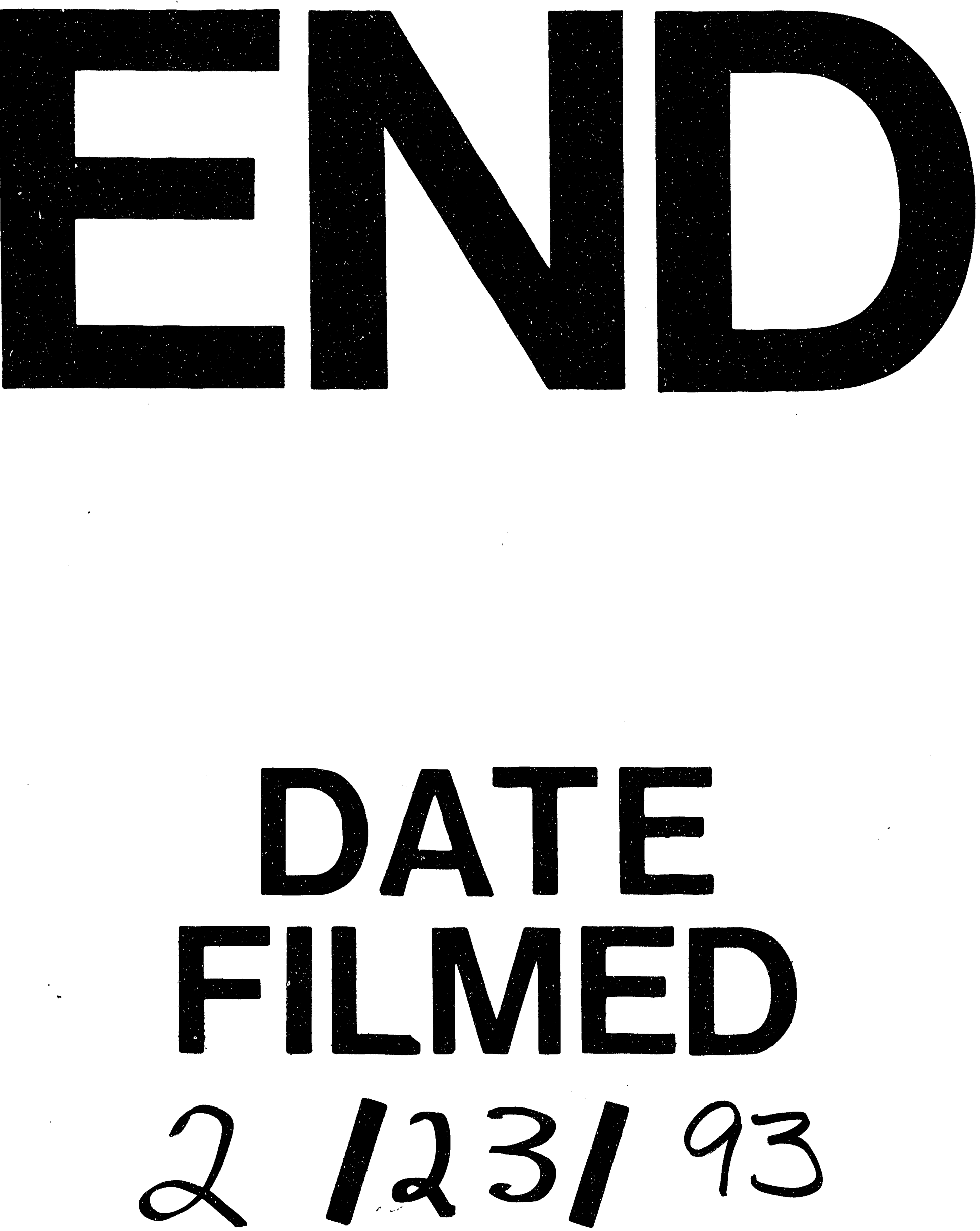
